\title{
Associations between beverage consumption of parents and their children. The ToyBox-study
}

\author{
Paloma Flores Barrantes ${ }^{1}$, Alicia Larruy ${ }^{1,2}$, Maria Luisa Miguel-Berges ${ }^{1,2}$, Pilar De \\ Miguel-Etayo $^{1,2}$, Iris Iglesia-Altaba ${ }^{1,3}$, Greet Cardon ${ }^{4}$, V. Iotova ${ }^{5}$, Koletzko Berthold ${ }^{6}$,

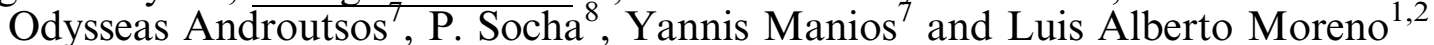 \\ ${ }^{1}$ GENUD Research Group, Zaragoza, Spain, \\ ${ }^{2}$ CIBER Fisiopatología de la Obesidad y Nutrición (CIBERobn), Instituto de Salud Carlos III, Madrid, Spain, \\ ${ }^{3}$ Red de Salud Materno-Infantil y del Desarrollo (SAMID), Madrid, Spain, \\ ${ }^{4}$ Department of Movement ans Sports Sciencies, Ghent, Belgium, \\ ${ }^{5}$ Medical University, Varna, Bulgaria, \\ ${ }^{6}$ Dr. von Hauner Childréns Hospital, Munich, Germany, \\ ${ }^{7}$ Department of Nutrition and Dietetics, School of Health Science and Education, Harokopio, Greece and \\ ${ }^{8}$ The Childrens Memorial Health Institute, Warsaw, Poland
}

\begin{abstract}
Introduction: Dietary habits are established from a very young age. Parental role modeling is an important factor influencing the eating behavior of their children. Drinking behavior may have an impact in the development of childhood obesity. This study aimed to explore the correlations of core drinking beverages between parents and their children.

Materials and Methods: The present study included children of 3.5-5.5 years and their parents from the (Multifactorial evidence-based approach using behavioral models in understanding and promoting fun, healthy food, play and policy for the prevention of obesity in early childhood) ToyBox study. The study was a kindergarten-based family-involved intervention, in preschool children from six European countries: Belgium, Bulgaria, Germany, Greece, Poland, and Spain. For this study, we analyzed data from the baseline cross-sectional survey.

Data on consumption frequency of water, homemade or fresh fruit juice, prepacked fruit juice, light beverages and sugared sweetened beverages consumption from parents and their children was obtained via a validated food frequency questionnaire. Parents were given examples of serving sizes and asked about how to self-report their usual consumption per day or week. Beverage consumption of children was reported by their parents and information about frequency and portion sizes was collected. Body weight and height of children was measured and classified according to the International Obesity Task Force (IOTF). Bivariate correlations were performed to analyze beverage consumption (servings per day) in children and their parents.
\end{abstract}

Results: The studied sample included 5266 pairs of children (49.2\% girls) and parents (91.7\% women) that were included in the analysis.

Girls presented higher correlations in water $\left(\mathrm{r}^{2}=0,317\right)$ and sugar sweetened beverages $\left(\mathrm{r}^{2}=0,302\right)$, whereas boys, presented slightly higher correlations of light soft drinks $\left(\mathrm{r}^{2}=0,273\right)$, pure fruit juices $\left(\mathrm{r}^{2}=0,308\right)$ and prepacked fruit juices $\left(\mathrm{r}^{2}=0,324\right)$, all of them at $<$ 0.01 level of significance. Considering boys and girls together, a slightly higher significant correlation coefficients were found between children-parents' dyads with overweight/obesity compared to normal weight children-parents for sugar sweetened beverages $\left(\mathrm{r}^{2}=\right.$ $0,303)$ and light soft drinks $\left(r^{2}=0,396\right)$.

Discussion: Beverage consumption of children and their parents were found to be moderately correlated. Overweight children seem to have better correlations with their parents in relation of sugar sweetened beverages and light soft drinks. Parents should encourage a healthy beverage consumption for their own health and most important, because there are key role models to their children.

\section{Conflict of Interest}

There is no conflict of interest. 